\title{
Protein disulfide isomerase a multifunctional protein with multiple physiological roles
}

\author{
Hyder Ali Khan and Bulent Mutus* \\ Chemistry and Biochemistry Department, University of Windsor, Windsor, ON, Canada
}

\section{Edited by:}

Fernando Antunes, Universidade de Lisbon, Portugal

Reviewed by:

Joao Batista Teixeira Rocha,

Universidade Federal de Santa

Maria, Brazil

Laura De Gara, Università Campus

Bio-Medico di Roma, Italy

*Correspondence:

Bulent Mutus, Chemistry and

Biochemistry Department,

University of Windsor, 401 Sunset

Avenue, Windsor, ON N9B 3P4,

Canada

e-mail:mutusb@uwindsor.ca
Protein disulfide isomerase (PDI), is a member of the thioredoxin superfamily of redox proteins. PDI has three catalytic activities including, thiol-disulfide oxireductase, disulfide isomerase and redox-dependent chaperone. Originally, PDI was identified in the lumen of the endoplasmic reticulum and subsequently detected at additional locations, such as cell surfaces and the cytosol. This review will provide an overview of the recent advances in relating the structural features of PDI to its multiple catalytic roles as well as its physiological and pathophysiological functions related to redox regulation and protein folding.

Keywords: PDI, protein disulfide isomerase, chaperone, oxidoreductase, disulfides, endoplasmic reticulum, cell surface

\section{INTRODUCTION}

In 1963, microsomal preparations of rat liver were shown to reactivate reduced ribonuclease A (Goldberger et al., 1963). The enzyme that catalyzed this reaction was eventually identified as protein disulfide isomerase (PDI; EC 5.3.4.1). PDI is one of 20 proteins belonging to the PDI family (Kozlov et al., 2010). The proteins in this family all contain at least one domain with a thioredoxin-like fold $(\beta \alpha \beta \alpha \beta \alpha \beta \beta \alpha)$, but may vary in length and domain arrangement (Kozlov et al., 2010).

Edman and coworkers were able to determine the active site sequence of rat PDI as WCGHCK through sequencing of cDNA (Edman et al., 1985). This sequence was homologous to the active site of thioredoxin and was found to catalyze the reduction and isomerization of disulfide bonds and the oxidation of thiols (Figure 1) (Holmgren, 1968). In 1994 Cai and coworkers discovered PDI also had chaperone activity in addition to its redox activity (Cai et al., 1994). In 1998, McLaughlin and Bulleid showed that the chaperone activity was independent of the redox status of active site thiols (McLaughlin and Bulleid, 1998). The three catalytic activities of PDI, thiol redox, disulfide exchange, and chaperone are central to endoplasmic reticulum (ER) function (Maattanen et al., 2010).

PDI is expressed in almost all mammalian tissues (Marcus et al., 1996; Noiva, 1999). Although PDI has a C-terminal KDEL ER retention sequence, significant amounts of this protein were shown to escape the ER and were detected in the nucleus, cytosol, cell surface, and extracellularly (Edman et al., 1985; Koch, 1987; Yoshimori et al., 1990).

This review will focus on recent discoveries on PDI structurefunction and physiological and pathophysiological roles arising from its localization in tissues including hemostasis, facilitation of pathogen entry, and reactive nitrogen and oxygen signaling. For more information on the role PDI in cancer, lipid homeostasis, infertility etc., the readers are directed to the reviews by Bulleid and Ellgaard (2011), Andreu et al. (2012), Laurindo et al. (2012), and Benham (2012).

\section{PDI STRUCTURE}

All members of the PDI family share the thioredoxin-like domain structure characterized by the $\beta \alpha \beta \alpha \beta \alpha \beta \beta \alpha$ fold (Kemmink et al., 1997). PDI contains four thioredoxin-like domains $\boldsymbol{a} \boldsymbol{b} \boldsymbol{b}^{\prime} \boldsymbol{a}$ '. The two redox active sites containing the CXXC motif are found in the $\boldsymbol{a}$ and $\boldsymbol{a}^{\prime}$ domains (Figure 2). The active site domains are linked by the $\boldsymbol{b}$ and $\boldsymbol{b}$ ' domains. There is also a small interdomain region known as the $\mathbf{x}$-linker located between $\boldsymbol{b}$ ' and $\boldsymbol{a}^{\prime}$ (Freedman et al., 1998; Alanen et al., 2003). For an in depth review on the structure of PDI the reader is directed to a recent review by Kozlov et al. (2010).

Recent advances in NMR and x-ray crystallography have given further insight into PDI structure and function, by identifying the $\boldsymbol{b}$ ' as the chaperone domain. Through NMR, the structure and amino acid residues of the $\boldsymbol{b}$ ' domain were observed to interact with unfolded RNase A, an oft used enzyme to assay the chaperone activity of PDI. The $\boldsymbol{b}$ ' domain contains a large multivalent hydrophobic surface allowing for a structurally promiscuous binding site (Denisov et al., 2009). In addition, computational analysis indicates that the $\boldsymbol{b} \boldsymbol{b}$ ' domains contain 4 cavities allowing for the possible binding of a variety of ligands (see Section PDI Chaperone Activity). Recently human PDI is observed to dimerize in vivo through the binding of $\boldsymbol{b} \boldsymbol{b}$ ' (Bastos-Aristizabal et al., 2014).

\section{PDI CHAPERONE ACTIVITY}

The chaperone activity of PDI is an essential area of study to further understand the protein folding related roles of PDI in the ER as well as neuronal tissues (see Section PDI and Coagulation). 


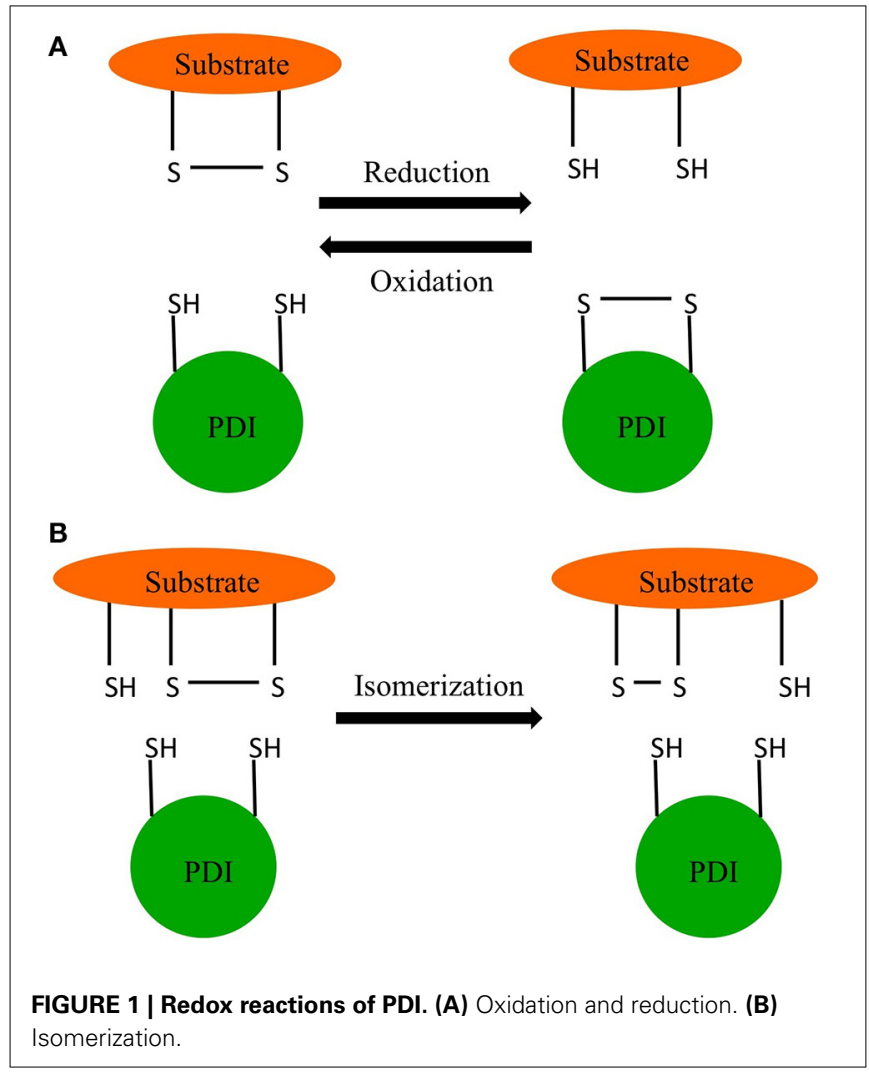

Historically the chaperone activity of PDI was assessed by a variety of methods depending on renaturation of denatured proteins monitored by activity-gain or loss-of-aggregates (Shao et al., 2000; Ben Khalaf et al., 2012; Wang et al., 2012; Hashimoto and Imaoka, 2013). A recent addition to this list utilizes aciddenatured green fluorescent protein (GFP), which interacts with a chaperone protein like PDI, and refold to yield the proper configuration and fluorescent properties (Mares et al., 2011). This technology allows for a high-throughput assays for chaperone activity and its inhibitors (Mares et al., 2011).

Recent work using NMR, indicated that PDI is able to distinguish between unfolded, partly folded, and fully folded proteins. In these studies, it was observed that the dissociation constant $\left(\mathrm{K}_{\mathrm{D}}\right)$ for fully unfolded basic pancreatic trypsin inhibitor was $\sim 1.5 \mu \mathrm{M}$ (Irvine et al., 2014). On the other hand the fully folded protein had a $K_{D}$ that was $\sim 10$ fold higher. Partially unfolded protein had a $K_{D}$ that was $\sim 3$ fold higher. These data lead the authors to conclude that PDI can distinguish between unfolded, partially unfolded, and folded proteins (Irvine et al., 2014).

A major recent discovery shows that the chaperone activity of PDI is regulated by its redox status. Wang and coworkers were the first to obtain the crystal structure of human PDI in both the reduced and oxidized forms (Wang et al., 2012). In oxidized form of PDI the active site of $\boldsymbol{a}$ and $\boldsymbol{a}$ ' are $40.3 \AA$ apart and the thioredoxin domains $\boldsymbol{a} \boldsymbol{b} \boldsymbol{b}$ ' $\boldsymbol{a}$ ' were all in the same plane (Wang et al., 2013). In the reduced state of PDI the active sites are $27.6 \AA$, however only $\boldsymbol{a} \boldsymbol{b} \boldsymbol{b}$ ' are in the same plane where $\boldsymbol{a}$ ' is twisted $45^{\circ}$ (Figure 2) (Wang et al., 2013), illustrating that the oxidized state has a more open conformation allowing for the entry of chaperone substrates (i.e., unfolded peptides) and the reduced state has a closed conformation inhibiting their entry. This further illustrates long range conformational changes induced by redox status of the active sites and further suggests redox regulation of chaperone activity.

Another interesting observation came from the work of $\mathrm{Fu}$ et al., where the hormone $17 \beta$-estradiol was able to bind to the hydrophobic cavity formed between the $\boldsymbol{b} \boldsymbol{b}$ ' domains (Fu et al., 2011). It should be noted that this site is different from the putative chaperone binding site in the $\boldsymbol{b}$ ' domain. The key amino acid residue in the interaction of PDI with $17 \beta$-estradiol was shown to $\mathrm{H} 256$ where it is believed that nitrogen of the histidine forms a hydrogen bond with the 3-hydroxyl group (Fu et al., 2011). The authors, based on this data, postulate yet another physiological role for PDI: a reservoir for hormones (Fu et al., 2011).

\section{PDI REDOX ACTIVITY AND ENDOPLASMIC RETICULUM OXIDOREDUCTIN-1}

When PDI catalyzes the oxidation of thiols and the reduction and isomerization of disulfides, the catalytic vicinal active site thiols (-CXXC-) undergo sequential oxidation and reduction reactions. It is suggested that an oxidized $\boldsymbol{a}$-domain catalyzes the oxidation of the reduced substrates and in the process becomes reduced. The N-terminus cysteine (C53) on the catalytic motif reacts with the substrate to form the mixed heterodimer while the C-terminus cysteine (C56) releases the substrate (Walker and Gilbert, 1997). The $\boldsymbol{a}$-domain is then subsequently oxidized by the $\boldsymbol{a}$-domain back to a disulfide through intramolecular reactions (Araki et al., 2013). The re-oxidation of the $\boldsymbol{a}$ '-domain is catalyzed by the protein endoplasmic reticulum oxidoreductin1 (Erol) in the process reducing $\mathrm{O}_{2}$ to produce $\mathrm{H}_{2} \mathrm{O}_{2}$ (Araki and Nagata, 2011). It should be noted that this enzyme has two isoforms $\alpha$ and $\beta$ (Wang et al., 2011). The binding affinity and catalytic activity of Ero1 $\alpha$ with PDI was observed to be better at $\mathrm{pH} 7$, and less so at 7.5 and 8 (Araki and Nagata, 2011). The interaction of Erol $\alpha$ with PDI occurs through the interaction of the $\beta$ hairpin in Erol $\alpha$ with the $\boldsymbol{b}$ '-domain of reduced PDI only (Masui et al., 2011). The non-covalent interaction was found to be between the aromatic residues, the W272 of Erol $\alpha$ and F240, F249, and F304 of PDI (Masui et al., 2011). This interaction allows for Erol $\alpha$ to be positioned in a manner that allows the oxidation of the $\boldsymbol{a}$-domain of PDI but not the $\boldsymbol{a}$-domain (Araki and Nagata, 2011; Masui et al., 2011). NMR studies on Ero1 $\alpha$ indicate that it is inactivated when disulfides are formed between C94-C104 and C99-C131 (Araki and Nagata, 2011). This conclusion was reached since C99 is the residue responsible for oxidizing the $\boldsymbol{a}$ '-domain of PDI. Therefore, it is thought the formation of disulfide between C94 and C104 results in the C99 close C131. The subsequent formation of the disulfide C99-C131 thus inactivates Ero1 $\alpha$ (Araki et al., 2013). The other isoform, Ero1 $\beta$ was observed to also preferentially oxidize the $\boldsymbol{a}$ ' domain of PDI (Nguyen et al., 2011; Wang et al., 2011).

A further consequence of Erol activity is the oxidation of the $\boldsymbol{a}$-domain of PDI. This is modulated through three other ER resident proteins: peroxiredoxin 4 (Prx IV), glutathione peroxidase 7 (GPx7), and glutathione peroxidase 8 (GPx8) (Kakihana et al., 2013; Wang et al., 2014). GPx7 and GPx8 catalyzes the reduction 


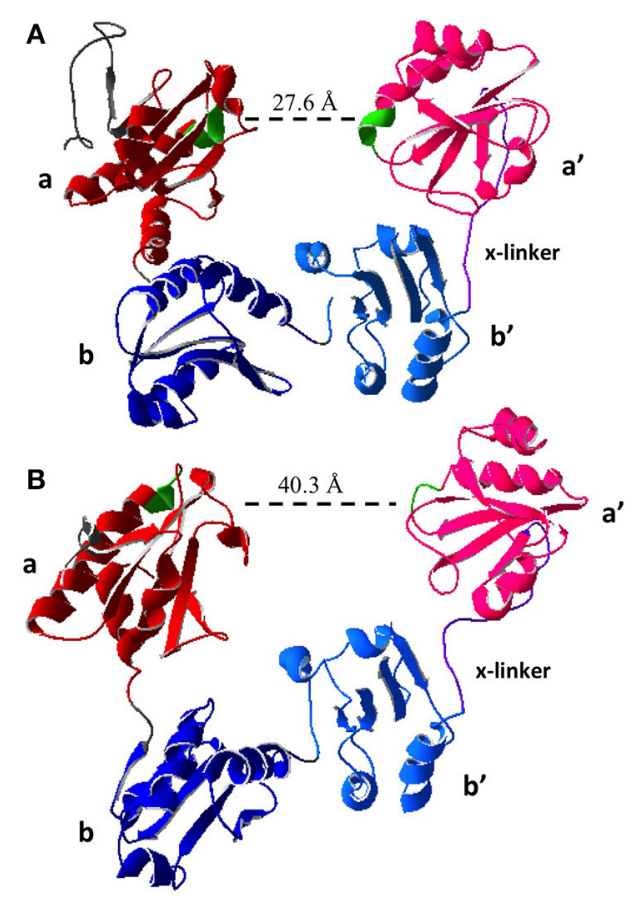

C

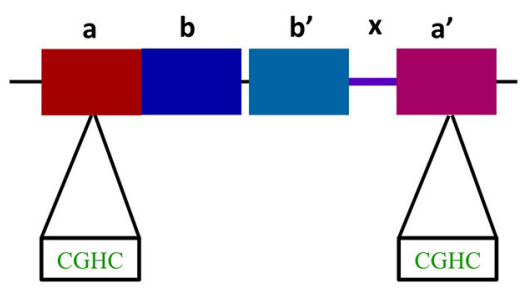

FIGURE 2 | Crystal Structure of Reduced and Oxidized PDI. (A) Reduced crystal structure of human PDI (PDB ID: 4EKZ). (B) Oxidized crystal structure of human PDI (PDB ID: 4EL1). (C) Schematic of PDI domains present in the crystal structure, as well as the two active site CXXC motifs (Crystal structures created using Swiss PDBViewer) (Wang et al., 2013). of Ero1-produced $\mathrm{H}_{2} \mathrm{O}_{2}$ to water. In the process, the $\mathrm{C} 57$ and C86 of GPx7 are converted to sulfenic acid and disulfide respectively. The PDI $\boldsymbol{a}$-domain then reduces these thiols, becoming oxidized in the process (Wang et al., 2014). When GPx7 or GPx8 was replaced with Prx IV this caused a slower oxidative folding of protein in comparison with either GPx7 or GPx8 (Nguyen et al., 2011). It is still controversial whether proteins such as, Erol $\alpha$, are the primary cause of the oxidation of PDI, since oxidized glutathione (GSSG) can also oxidize PDI active sites (Lappi and Ruddock, 2011). The second-order rate constant for the formation of mixed disulfide from reduced PDI and GSSG was observed to be $191 \pm 3 \mathrm{M}^{-1} \mathrm{~s}^{-1}$ (Lappi and Ruddock, 2011). In vivo studies of the rate of oxidation of the $\boldsymbol{a}$ domain of PDI by GSSG were found to be $\sim 0.3 \mathrm{~s}^{-1}$, indicating that this rate is too fast to be overlooked (Lappi and Ruddock, 2011). The oxidation of PDI by excess $\mathrm{H}_{2} \mathrm{O}_{2}$ is also observed and has a pseudo-first-order reaction with a rate constant of $9.2 \mathrm{M}^{-1} \mathrm{~s}^{-1}$ (Karala et al., 2009). However this study was conducted solely on the $\boldsymbol{a}$ domain on PDI, further studies of glutathione and glutathione disulfide on the oxidation of PDI are required to determine how much affect these molecules have on PDI. It should also be noted that more research must be conducted on how much affect PDI has on controlling the equilibrium of glutathione and glutathione disulfide.

\section{PDI INHIBITORS}

With the importance of PDI in many different cellular functions, there has been an increased interest in the modulation of its activity with small molecule inhibitors. A commonly used inhibitor of PDI is bacitracin, which is a mixture of cyclic polypeptides.
Recently, the analogs of bacitracin were individually tested to observe their inhibitory effect on the reductive activity of PDI. The analogs $\mathrm{H}$ and $\mathrm{F}$ were 25 -fold more effective than the $\mathrm{A}$ and B analogs (Dickerhof et al., 2011). These analogs bind to the b' domain and interestingly do not seem to inhibit the oxidative and isomerase activity of PDI (Roth, 1981; Dickerhof et al., 2011).

Another inhibitor of PDI reductase activity is quercetin-3rutinoside. This compound inhibits PDI mediated platelet aggregation as a result has been suggested as antithrombic agent (Jasuja et al., 2012). Phenyl vinyl sulfonate containing molecules were also showed to inhibit PDI (Ge et al., 2013). The inhibitor designated P1 was observed to inhibit PDI in mammalian cells with an $\mathrm{IC}_{50}$ of $1.7 \pm 0.4 \mu \mathrm{m}$ (Ge et al., 2013).

Propynoic acid carbamoyl methyl amides (PACMA) also inhibit PDI (Xu et al., 2012). Of these, the one designated PACMA31 was observed to be an irreversible inhibitor, by forming a covalent (carbon-sulfur) bond to the active site cysteine (Xu et al., 2012). Intriguingly, this inhibitor was observed to target ovarian tumors, inhibiting their growth but not causing harm to normal cells (Xu et al., 2012).

\section{PDI AND NADPH OXIDASE}

NADPH oxidase complex (Nox) is the major contributor of reactive oxygen species (ROS) in cells which can act as molecular signal and when produced in excess can cause oxidative stress (Janiszewski et al., 2005; Clempus and Griendling, 2006; Bedard and Krause, 2007; Marchetti et al., 2007). The NADPH oxidase complex is comprised of many subunits, that are expressed differently depending on the cell type (Janiszewski et al., 2005; Clempus 
and Griendling, 2006; Bedard and Krause, 2007; Marchetti et al., 2007). Laurindo's group were the first to report a role for PDI in the regulation of Nox. PDI is shown to interact with Nox within the ER as well as outside the ER in the cytosol (Laurindo et al., 2008, 2014; Santos et al., 2009b).

More recently, Fernandes et al. showed that in vascular smooth muscle cells, the overexpression of PDI increased Nox1 mRNA which encodes for NOX1 in NADPH oxidase complex (Fernandes et al., 2009). The PDI overexpression also produced an increase in the activity of NADPH oxidase, leading to an increase in the steady-state levels of ROS (Fernandes et al., 2009). Silencing of PDI triggered a decrease in mRNA and protein of Nox1 (Fernandes et al., 2009). Overexpression of a mutant PDI where the catalytic cysteines were mutated resulted in the increase in Nox1 mRNA and protein indicating that PDI redox activity is not required and it is most likely the chaperone activity of PDI that is modulating this effect (Fernandes et al., 2009).

In other studies, PDI silencing has shown to greatly decrease platelet derived growth factor (PDGF)-induced VSMC migration (Pescatore et al., 2012). In the same study, it was observed that PDI co-immunoprecipitated with Rac1, RhoA, and RhoGDI (Pescatore et al., 2012). In macrophages, PDI plays a similar role where interaction with NADPH oxidase causes an increase in ROS (Santos et al., 2009a). Laurindo's group has shown that PDI coimmuneprecipitated and co-localized with p22phox subunit of NADPH oxidase in J774 cells (Santos et al., 2009a). They also used a leukocyte cell free system to observe the interaction of PDI with NADPH oxidase. They found that oxidized PDI stimulates ROS production however reduced PDI inhibited the production of ROS (De et al., 2011). This group further suggests that PDI possibly interacts with p47phox subunit of NADPH oxidase through hydrophobic effects and not through cysteines (Santos et al., 2009a). From all this data it can be suggested that PDI plays an important role in the regulation of ROS.

\section{PDI AND INFECTION}

The internalization of some pathogens has been shown to be modulated by PDI. Ryser and coworkers were the first to test the potential role of PDI in the internalization of pathogens (Ryser et al., 1994). In mouse macrophage J774 cells, Laurindo's group observed that increased levels of PDI would increase the phagocytosis of $L$. chagasi promastigote but not the amastigote (Santos et al., 2009a). Phagocytosis was decreased by inhibition of expression levels of PDI as well as through the addition of thiol inhibitors such as p-phenylarsine oxide (Santos et al., 2009a). Santos and coworkers hypothesized that PDI plays a role in the reduction of the disulfide bonds present on the parasite, which may help with internalization of the parasite (Santos et al., 2009a). They also suggest that PDI's role in the increase of ROS through the NADPH oxidase interaction resulting in an "intraphagosomal-oxidizing milieu" and thus favoring infection of promastigotes (Santos et al., 2009a).

Reiser and coworkers have examined the role of PDI in HIV infections. Both PDI and thioredoxin $1(\operatorname{Trx} 1)$ have been shown to reduce disulfides on the viral glycoprotein gp120 causing the internalization of HIV-1 (Reiser et al., 2012). Through semiquantitative ELISA analysis it was observed that Trx1 was more efficient at reducing disulfides on gp120, however the authors hypothesize that the two proteins may be acting at different stages of internalization (Reiser et al., 2012). Interestingly, galectin-9 was observed to bind to PDI on Th2 cells this helped with the retention of PDI to the cell surface as well as increased the entry of HIV into these cells (Bi et al., 2011). It is suggested that inhibitors of gp120 that prevent the reduction of the disulfides maybe a better drug target than reductases since reductases are widely used in cells (Reiser et al., 2012).

In endothelial cells surface PDI possibly reduces $\beta 1$ and $\beta 3$ integrins allowing for the entry of dengue virus (Wan et al., 2012). Also in MA104 cells thiol blockers and PDI inhibitors decreased the entry of rotavirus, indicating the involvement of thiols for infectivity (Calderon et al., 2012).

Recently, PDI was observed to regulate the cytoskeleton reorganization by its interaction with $\beta$-actin (Sobierajska et al., 2014). PDI was observed to bind to Cys374 of $\beta$-actin through a disulfide bond (Sobierajska et al., 2014). In MEG-01 cells the down regulation of PDI resulted in the prevention of adhesion to fibronectin (Sobierajska et al., 2014).

During the bacterial infection of the cholera toxin it is observed that PDI does not unfold the CT protein into its subunit CTA1 for further infection (Taylor et al., 2011). Nevertheless, PDI does disassemble the holotoxin allowing for the dissociation of CTA1 from CTA2 and $\mathrm{CTB}_{5}$ and its spontaneous unfolding, allowing CTA1 to exit the ER (Taylor et al., 2011). Interestingly, Taylor and coworkers recently discovered that PDI when interacting with the holotoxin, will itself unfold, causing a wedge to form between the holotoxin and causing the dissociation of CTA1 (Taylor et al., 2014). PDI locked in the folded state would not dissociate the holotoxin (Taylor et al., 2014). This appears not to required the oxireductase activity of PDI since redox inhibitors had no effect on toxin activation. On the other hand, chaperone inhibitors prevent the release of CTA1 thus indicating that PDI unfolding in the presence of holotoxin requires PDI chaperone activity (Taylor et al., 2014).

\section{PDI AND COAGULATION}

The activation of platelets is a complex series of proteolytic, protein-protein, and protein-ligand interactions that is not yet fully understood. A quick summary of this process is, that damaged endothelial cells will cause the exposure of collagen allowing for the glycoprotein, von Willebrand factor to attract platelets (Lopez et al., 1992; Modderman et al., 1992; Ruggeri, 2007; Herr and Farndale, 2009). The platelets form a monolayer, which in turns produces thrombin for additional platelet aggregation and causes a series of cascades (Monroe et al., 2002; Clemetson, 2012). The platelets also start to secrete other activators these will bind to receptor on the platelets and increase the intracellular calcium levels (Purvis et al., 2008). This causes receptors on platelets to bind fibrinogen resulting in the increase adhesion of the platelets and the formation of thrombi(Ma et al., 2007; Furie and Furie, 2008). Many of the integrin receptors associated with platelet activation contain a cysteine rich subunit, possibly allowing for PDI to regulate the redox state of the thiols.

Platelets deficient of PDI display irregular and improper aggregation, when wild-type PDI is added the aggregation of platelets 
returns to normal (Kim et al., 2013). PDI on the surface of platelets plays a crucial role in the formation of thrombus in collagen-coated platelets (Kim et al., 2013). In monocytic cells, cell surface PDI is required for antithymocyte globulin decryption of tissue factor (Langer et al., 2013).

Recent studies have shown that free thiols and isomerization are associated with the process of coagulation (Jurk et al., 2011). In vitro studies by Jurk and coworkers have shown that PDI plays a role in the feedback activation of thrombin in platelets that have already been stimulated by thrombin (Jurk et al., 2011). In the same paper, they showed that PDI may play a role in the interaction of coagulation factors with platelets (Jurk et al., 2011). With the use of $\mathrm{CHO}$ cells expressing different integrin factors, $\alpha_{V} \beta_{3}$ and $\alpha_{\mathrm{IIb}} \beta_{3}$,PDI was observed to bind to these integrin molecules (Cho et al., 2012). This binding of PDI with the integrin was determined to be through the $\beta_{3}$ subunit and not the $\alpha$ subunit (Cho et al., 2012). The binding of PDI to integrins was not inhibited by the presence of PDI redox inhibitors, the authors however do not hypothesize how this interaction is occurring (Cho et al., 2012). In a mice model, where there is a deficiency in $\beta_{3}$ integrins, it is observed that fibrin generation was diminished from the lack of accumulation of PDI at the platelet surface (Cho et al., 2012). In neutrophil cells, PDI may mediate the adhesive activity during vasculature inflammation through its interaction with $\alpha_{M} \beta_{2}$ integrin (Hahm et al., 2013). With the use of flow cytometry, it was observed that exogenous PDI interacted with neutrophil cell surface by electrostatic interactions (Hahm et al., 2013). The authors propose that PDI catalyzes thiol exchange through electrostatic interactions of $\alpha_{M} \beta_{2}$ integrin thus regulating the clustering of the integrin within lipid rafts (Hahm et al., 2013). It should also be noted that vitamin $\mathrm{K}$ epoxide reductase, an important enzyme in blood coagulation, requires the help of a thioredoxin-like protein to regenerate vitamin $\mathrm{K}$ hydroquinone from the epoxide form. It is speculated that the PDI is the thioredoxin-like protein that provides the reductive equivalent to perform the regeneration (Schulman et al., 2010).

In endothelial cells, when PDI was silenced or inhibited on the cell surface, the coagulation was increased (Popescu et al., 2010). In agreement with this finding, it was observed that addition of exogenous PDI resulted in the decrease in coagulation activity (Popescu et al., 2010). The authors suggest that coagulation of endothelial cells is negatively regulated by PDI, specifically its oxidoreductase activity (Popescu et al., 2010). In the same study, it was also observed that PDI may play a role in the regulation of phosphatidylserine exposure through the activity of flippase and floppase (Popescu et al., 2010).

For more information of previous knowledge on the effects of redox on coagulation can be found in reviews by Cho (2013), Flaumenhaft (2013), Langer and Ruf (2014), and Murphy et al. (2014).

\section{PDI AND SUPEROXIDE DISMUTASE 1}

Protein aggregation has been shown to occur in a variety of neurodegenerative diseases and disorders, such as cerebral ischemia and amyotrophic lateral sclerosis. Cai and coworkers were one of the first to show that PDI alleviates the aggregation of proteins (Cai et al., 1994). Cerebral ischemia is caused by decrease blood flow to the brain resulting in the loss of oxygen to neurons and astrocytes, which has shown to cause an increase in nitric oxide (NO) production specifically by inducible nitric oxide synthase (iNOS) (Cherian et al., 2000; Greve and Zink, 2009). Elevated NO production can lead to the inactivation of proteins by $S$-nitrosylation. In astrocytes, recent studies by Chen have shown the presence of S-nitrosylated PDI (SNO-PDI), which has previously been shown to inhibit the activity of PDI (Ramachandran et al., 2001; Chen et al., 2012). When the cells were oxygen deprived, this caused the formation of ubiquitinated protein aggregates, of proteins like superoxide dismutase 1 (SOD1), which is crucial to cell survival (Chen et al., 2012).

When NO production in these cells was attenuated, the formation of both SNO-PDI and protein aggregates decreased (Chen et al., 2012). Interestingly, in SH-SY5Y cells the overexpression of mutant SOD1 caused an increase expression of iNOS, where wild type SOD1 did not cause any increase (Chen et al., 2013). SOD1 is a protein that alleviates oxidative stress by converting free radicals to $\mathrm{H}_{2} \mathrm{O}_{2}$ (Chen et al., 2012). The mutant SOD1 expressing cells were observed to contain SNO-PDI and protein aggregation while the wild type SOD1 did not show any increase in either, this was also observed in vivo, in transgenic mice (Chen et al., 2013). Furthermore, the expression of PDI increased when protein aggregates accumulated in cells, and the cells became apoptotic (Hoffstrom et al., 2010; Chen et al., 2013).

The aggregation of mutant SOD1 has shown to cause an increase in neuronal cell death in amyotrophic lateral sclerosis, it has been hypothesized that denitrosylation of PDI can be considered as a therapeutic intervention for this disease (Jeon et al., 2014). In both HEK293A cells and NSC-34 motor neuron cells, the overexpression of PDI lead to a decrease in the aggregation of mutant SOD1 (Jeon et al., 2014). The interaction of PDI with SOD1 appears to be through the chaperone activity of PDI rather than the isomerase activity since aggregation was also reduced with mutant PDI lacking active site thiols (Jeon et al., 2014). Intriguingly, the overexpression of PDI caused by mutant SOD1 has shown to cause the activation of NADPH oxidase causing an increase in ROS (Jaronen et al., 2013). In murine microglial BV-2 cells Nox activation was PDI-dependent suggesting that PDI inhibitors may help reduce oxidative stress in cells (Jaronen et al., 2013). However, further studies must be conducted to determine PDI's role in the interplay of protein aggregation and the production of ROS.

\section{ROLE OF PDI IN NITRIC OXIDE SIGNALING}

Our group was the first to show that cell surface PDI mediated the entry of $S$-nitrosothiols (SNO) into cells (Ramachandran et al., 2001). Cell surface PDI was observed to release NO from $S$-nitrosylated glutathione (GSNO), one of the main nitric oxide carriers in tissues (Root et al., 2004). PDI would denitrosylate GSNO when NO levels were low, however during high levels of $\mathrm{NO}$, PDI would act as a carrier either through the formation of SNO-PDI (Sliskovic et al., 2005).

Recently our group, showed that PDI plays a role in the transport of nitric oxide from red blood cells to endothelium cells (Kallakunta et al., 2013). This process was observed to be oxygen-dependent where nitric oxide bound hemoglobin 


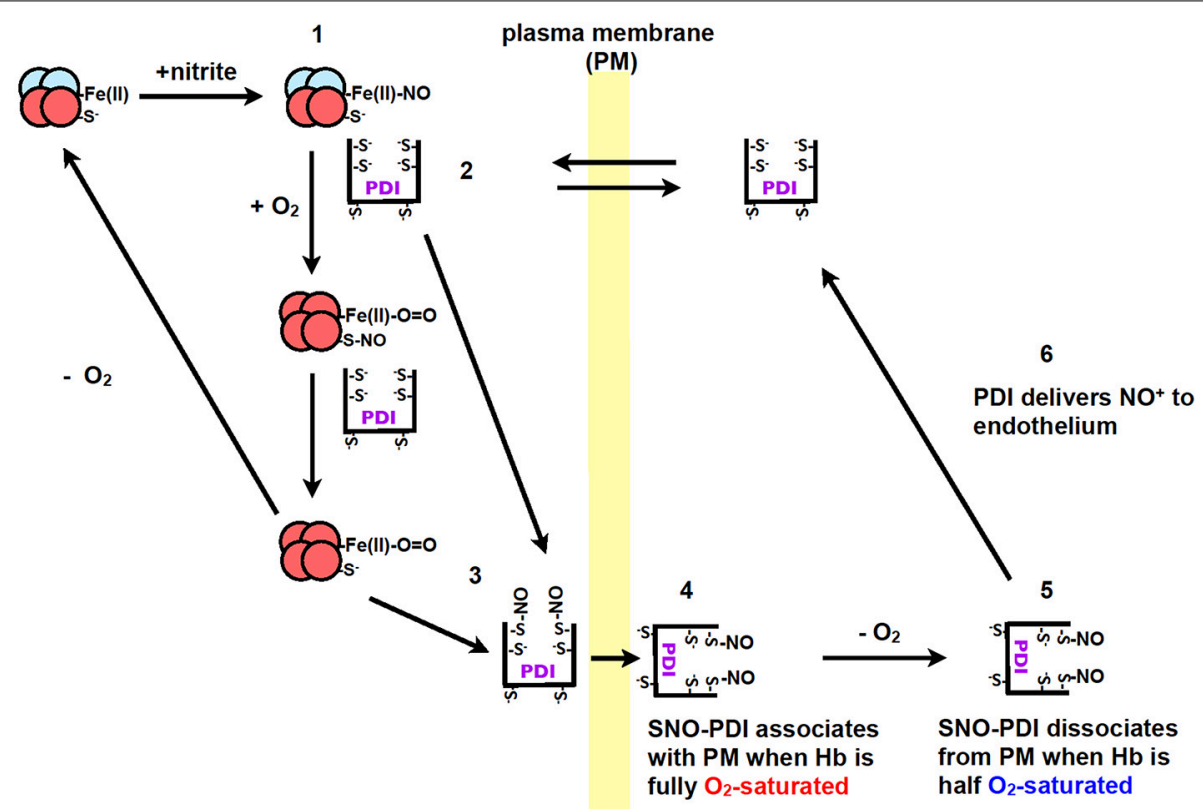

FIGURE 3 | The formation of $\mathrm{NO}^{+} / \mathrm{NO}$ from nitrite during hypoxic conditions by $\mathrm{Hb}$ and the transfer of nitric oxide to endothelium by PDI. (1) Nitrite reacts with $\mathrm{Hb}$ under hypoxic conditions to form Fe(II)-NO. (2) PDI from blood equilibrates across the plasma membrane of red blood cells to form a complex with $\mathrm{Hb}$. (3) When red blood cells enter the lungs the $\mathrm{O}_{2}$ displaces the NO from the iron on the heme group to PDI thiols or to $\mathrm{Hb}$ (Cys $\beta 93)$, resulting in the formation of SNO-PDI. (4) PDI attaches to the extracellular surface of red blood cells under normoxic conditions. (5) When the red blood cells enter tissue that is under hypoxic conditions, SNO-PDI is released. (6) SNO-PDI interacts with the endothelium cells, releasing $\mathrm{NO}^{+} / \mathrm{NO}$ triggering hypoxic vasodilation. Image taken from Kallakunta et al. (2013).
$(\mathrm{NO}-\mathrm{Hb})$ required hypoxic conditions. Oxygen is then required for the formation of SNO-Hb, which then transfers the nitric oxide to PDI to form SNO-PDI (Kallakunta et al., 2013). When hemoglobin is half oxygen saturated it was observed that nitric oxide would dissociate from extracellular PDI and be transferred to endothelium cells (Figure 3) (Kallakunta et al., 2013).

\section{CONCLUSION}

During the early years of research on PDI it was well thought of that PDI was required for the maintenance of healthy cells and tissues, in view of the catalytic roles of PDI in reducing and isomerizing disulfides into native confirmations of proteins and to alleviate protein aggregates through its chaperone activity. However recent studies indicate that this may not be the case. PDI through its thiol redox activity, is observed to play a role for the internalization of some pathogens, as well as it is shown to promote increased ROS in cells. Furthermore, in neuronal cells post transnationally modified SNO-PDI etc. has been shown to promote protein aggregation commonly associated with the pathophysiology of neurodegenerative diseases. These observations show that PDI is a complex protein that can play a role in both physiology and pathophysiology. Therefore, further studies are warranted in order to solve the complex relationships of PDI in health and disease.

\section{ACKNOWLEDGMENT}

This work was supported by a NSERC Discovery Grant to Bulent Mutus and was supported by the University of Windsor tuition scholarship.

\section{REFERENCES}

Alanen, H. I., Salo, K. E., Pekkala, M., Siekkinen, H. M., Pirneskoski, A., and Ruddock, L. W. (2003). Defining the domain boundaries of the human protein disulfide isomerases. Antioxid. Redox Signal. 5, 367-374. doi: $10.1089 / 152308603768295096$

Andreu, C. I., Woehlbier, U., Torres, M., and Hetz, C. (2012). Protein disulfide isomerases in neurodegeneration: from disease mechanisms to biomedical applications. FEBS Lett. 586, 2826-2834. doi: 10.1016/j.febslet.2012. 07.023

Araki, K., Iemura, S., Kamiya, Y., Ron, D., Kato, K., Natsume, T., et al. (2013). Ero1-alpha and PDIs constitute a hierarchical electron transfer network of endoplasmic reticulum oxidoreductases. J. Cell Biol. 202, 861-874. doi: $10.1083 /$ jcb.201303027

Araki, K., and Nagata, K. (2011). Functional in vitro analysis of the ERO1 protein and protein-disulfide isomerase pathway. J. Biol. Chem. 286, 32705-32712. doi: 10.1074/jbc.M111.227181

Bastos-Aristizabal, S., Kozlov, G., and Gehring, K. (2014). Structural insight into the dimerization of human protein disulfide isomerase. Protein Sci. 23, 618-626. doi: $10.1002 /$ pro. 2444

Bedard, K., and Krause, K.-H. (2007). The NOX family of ROS-generating NADPH oxidases: physiology and pathophysiology. Physiol. Rev. 87, 245-313. doi: 10.1152/physrev.00044.2005

Benham, A. M. (2012). The protein disulfide isomerase family: key players in health and disease. Antioxid. Redox Signal. 16, 781-789. doi: 10.1089/ars. 2011.4439 
Ben Khalaf, N., De Muylder, G., Louzir, H., Mckerrow, J., and Chenik, M. (2012). Leishmania major protein disulfide isomerase as a drug target: enzymatic and functional characterization. Parasitol. Res. 110, 1911-1917. doi: 10.1007/s00436-011-2717-5

Bi, S., Hong, P. W., Lee, B., and Baum, L. G. (2011). Galectin-9 binding to cell surface protein disulfide isomerase regulates the redox environment to enhance T-cell migration and HIV entry. Proc. Natl. Acad. Sci. U.S.A. 108, 10650-10655. doi: $10.1073 /$ pnas.1017954108

Bulleid, N. J., and Ellgaard, L. (2011). Multiple ways to make disulfides. Trends Biochem. Sci. 36, 485-492. doi: 10.1016/j.tibs.2011.05.004

Cai, H., Wang, C. C., and Tsou, C. L. (1994). Chaperone-like activity of protein disulfide isomerase in the refolding of a protein with no disulfide bonds. J. Biol. Chem. 269, 24550-24552.

Calderon, M. N., Guerrero, C. A., Acosta, O., Lopez, S., and Arias, C. F. (2012). Inhibiting rotavirus infection by membrane-impermeant thiol/disulfide exchange blockers and antibodies against protein disulfide isomerase Intervirology 55, 451-464. doi: 10.1159/000335262

Chen, X., Guan, T., Li, C., Shang, H., Cui, L., Li, X. M., et al. (2012). SOD1 aggregation in astrocytes following ischemia/reperfusion injury: a role of NO-mediated S-nitrosylation of protein disulfide isomerase (PDI). J. Neuroinflammation 9, 237. doi: 10.1186/1742-2094-9-237

Chen, X., Zhang, X., Li, C., Guan, T., Shang, H., Cui, L., et al. (2013). S-nitrosylated protein disulfide isomerase contributes to mutant SOD1 aggregates in amyotrophic lateral sclerosis. J. Neurochem. 124, 45-58. doi: 10.1111/jnc.12046

Cherian, L., Goodman, J. C., and Robertson, C. S. (2000). Brain nitric oxide changes after controlled cortical impact injury in rats. J. Neurophysiol. 83 , 2171-2178. doi: 10.1097/00003246-199901001-00091

Cho, J. (2013). Protein disulfide isomerase in thrombosis and vascular inflammation. J. Thromb. Haemost. 11, 2084-2091. doi: 10.1111/jth.12413

Cho, J., Kennedy, D. R., Lin, L., Huang, M., Merrill-Skoloff, G., Furie, B. C., et al. (2012). Protein disulfide isomerase capture during thrombus formation in vivo depends on the presence of beta 3 integrins. Blood 120, 647-655. doi: 10.1182/blood-2011-08-372532

Clemetson, K. J. (2012). Platelets and primary haemostasis. Thromb. Res. 129, 220-224. doi: 10.1016/j.thromres.2011.11.036

Clempus, R. E., and Griendling, K. K. (2006). Reactive oxygen species signaling in vascular smooth muscle cells. Cardiovasc. Res. 71, 216-225. doi: 10.1016/j.cardiores.2006.02.033

De, A. P.A. M., Verissimo-Filho, S., Guimaraes, L. L., Silva, A. C., Takiuti, J. T., and Lopes, L. R. (2011). Protein disulfide isomerase redox-dependent association with p47(phox): evidence for an organizer role in leukocyte NADPH oxidase activation. J. Leukoc. Biol. 90, 799-810. doi: 10.1189/jlb.0610324

Denisov, A. Y., Maattanen, P., Dabrowski, C., Kozlov, G., Thomas, D. Y., and Gehring, K. (2009). Solution structure of the bb' domains of human protein disulfide isomerase. FEBS J. 276, 1440-1449. doi: 10.1111/j.17424658.2009.06884.x

Dickerhof, N., Kleffmann, T., Jack, R., and Mccormick, S. (2011). Bacitracin inhibits the reductive activity of protein disulfide isomerase by disulfide bond formation with free cysteines in the substrate-binding domain. FEBS J. 278 , 2034-2043. doi: 10.1111/j.1742-4658.2011.08119.x

Edman, J. C., Ellis, L., Blacher, R. W., Roth, R. A., and Rutter, W. J. (1985). Sequence of protein disulphide isomerase and implications of its relationship to thioredoxin. Nature 317, 267-270.

Fernandes, D. C., Manoel, A. H., Wosniak, J. Jr., and Laurindo, F. R. (2009). Protein disulfide isomerase overexpression in vascular smooth muscle cells induces spontaneous preemptive NADPH oxidase activation and Nox1 mRNA expression: effects of nitrosothiol exposure. Arch. Biochem. Biophys. 484, 197-204. doi: 10.1016/j.abb.2009.01.022

Flaumenhaft, R. (2013). Protein disulfide isomerase as an antithrombotic target. Trends Cardiovasc. Med. 23, 264-268. doi: 10.1016/j.tcm.2013.03.001

Freedman, R. B., Gane, P. J., Hawkins, H. C., Hlodan, R., Mclaughlin, S. H., and Parry, J. W. (1998). Experimental and theoretical analyses of the domain architecture of mammalian protein disulphide-isomerase. Biol. Chem. 379, 321-328.

Fu, X. M., Wang, P., and Zhu, B. T. (2011). Characterization of the estradiol-binding site structure of human protein disulfide isomerase (PDI). PLoS ONE 6:e27185. doi: 10.1371/journal.pone. 0027185

Furie, B., and Furie, B. C. (2008). Mechanisms of thrombus formation. N. Engl. J. Med. 359, 938-949. doi: 10.1056/NEJMra0801082
Ge, J., Zhang, C. J., Li, L., Chong, L. M., Wu, X., Hao, P., et al. (2013). Small molecule probe suitable for in situ profiling and inhibition of protein disulfide isomerase. ACS Chem. Biol. 8, 2577-2585. doi: 10.1021/cb4002602

Goldberger, R. F., Epstein, C. J., and Anfinsen, C. B. (1963). Acceleration of reactivation of reduced bovine pancreatic ribonuclease by a microsomal system from rat liver. J. Biol. Chem. 238, 628-635.

Greve, M. W., and Zink, B. J. (2009). Pathophysiology of traumatic brain injury. Mt. Sinai J. Med. 76, 97-104. doi: 10.1002/msj.20104

Hahm, E., Li, J., Kim, K., Huh, S., Rogelj, S., and Cho, J. (2013). Extracellular protein disulfide isomerase regulates ligand-binding activity of alphaMbeta2 integrin and neutrophil recruitment during vascular inflammation. Blood 121, 3789-3800, S3781-S3715. doi: 10.1182/blood-2012-11-467985

Hashimoto, S., and Imaoka, S. (2013). Protein-disulfide isomerase regulates the thyroid hormone receptor-mediated gene expression via redox factor1 through thiol reduction-oxidation. J. Biol. Chem. 288, 1706-1716. doi: 10.1074/jbc.M112.365239

Herr, A. B., and Farndale, R. W. (2009). Structural insights into the interactions between platelet receptors and fibrillar collagen. J. Biol. Chem. 284, 19781-19785. doi: 10.1074/jbc.R109.013219

Hoffstrom, B. G., Kaplan, A., Letso, R., Schmid, R. S., Turmel, G. J., Lo, D. C., et al. (2010). Inhibitors of protein disulfide isomerase suppress apoptosis induced by misfolded proteins. Nat. Chem. Biol. 6, 900-906. doi: 10.1038/nchembio.467

Holmgren, A. (1968). Thioredoxin. 6. The amino acid sequence of the protein from Escherichia coli B. Eur. J. Biochem. 6, 475-484.

Irvine, A. G., Wallis, A. K., Sanghera, N., Rowe, M. L., Ruddock, L. W., Howard, M. J., et al. (2014). Protein disulfide-isomerase interacts with a substrate protein at all stages along its folding pathway. PLoS ONE 9:e82511. doi: 10.1371/journal.pone.0082511

Janiszewski, M., Lopes, L. R., Carmo, A. O., Pedro, M. A., Brandes, R. P., Santos, C. X., et al. (2005). Regulation of NAD(P)H oxidase by associated protein disulfide isomerase in vascular smooth muscle cells. J. Biol. Chem. 280, 40813-40819. doi: 10.1074/jbc.M509255200

Jaronen, M., Vehvilainen, P., Malm, T., Keksa-Goldsteine, V., Pollari, E., Valonen, P., et al. (2013). Protein disulfide isomerase in ALS mouse glia links protein misfolding with NADPH oxidase-catalyzed superoxide production. Hum. Mol. Genet. 22, 646-655. doi: 10.1093/hmg/dds472

Jasuja, R., Passam, F. H., Kennedy, D. R., Kim, S. H., Van Hessem, L., Lin, L., et al. (2012). Protein disulfide isomerase inhibitors constitute a new class of antithrombotic agents. J. Clin. Invest. 122, 2104-2113. doi: 10.1172/ JCI61228

Jeon, G. S., Nakamura, T., Lee, J.-S., Choi, W.-J., Ahn, S.-W., Lee, K.-W., et al. (2014). Potential effect of $S$-nitrosylated protein disulfide isomerase on mutant SOD1 aggregation and neuronal cell death in amyotrophic lateral sclerosis. Mol. Neurobiol. 49, 796-807. doi: 10.1007/s12035-013-8562-z

Jurk, K., Lahav, J., VAN Aken, H., Brodde, M. F., Nofer, J. R., and Kehrel, B. E. (2011). Extracellular protein disulfide isomerase regulates feedback activation of platelet thrombin generation via modulation of coagulation factor binding. J. Thromb. Haemost. 9, 2278-2290. doi: 10.1111/j.1538-7836.2011. 04509.x

Kakihana, T., Araki, K., Vavassori, S., Iemura, S., Cortini, M., Fagioli, C., et al. (2013). Dynamic regulation of Erolalpha and peroxiredoxin 4 localization in the secretory pathway. J. Biol. Chem. 288, 29586-29594. doi: 10.1074/jbc.M113.467845

Kallakunta, V. M., Slama-Schwok, A., and Mutus, B. (2013). Protein disulfide isomerase may facilitate the efflux of nitrite derived S-nitrosothiols from red blood cells. Redox Biol. 1, 373-380. doi: 10.1016/j.redox.2013.07.002

Karala, A. R., Lappi, A. K., Saaranen, M. J., and Ruddock, L. W. (2009). Efficient peroxide-mediated oxidative refolding of a protein at physiological $\mathrm{pH}$ and implications for oxidative folding in the endoplasmic reticulum. Antioxid. Redox Signal. 11, 963-970. doi: 10.1089/ARS.2008.2326

Kemmink, J., Darby, N. J., Dijkstra, K., Nilges, M., and Creighton, T. E. (1997). The folding catalyst protein disulfide isomerase is constructed of active and inactive thioredoxin modules. Curr. Biol. 7, 239-245.

Kim, K., Hahm, E., Li, J., Holbrook, L. M., Sasikumar, P., Stanley, R. G., et al. (2013). Platelet protein disulfide isomerase is required for thrombus formation but not for hemostasis in mice. Blood 122, 1052-1061. doi: 10.1182/blood-201303-492504

Koch, G. L. (1987). Reticuloplasmins: a novel group of proteins in the endoplasmic reticulum. J. Cell Sci. 87(Pt 4), 491-492. 
Kozlov, G., Maattanen, P., Thomas, D. Y., and Gehring, K. (2010). A structural overview of the PDI family of proteins. FEBS J. 277, 3924-3936. doi: 10.1111/j.1742-4658.2010.07793.x

Langer, F., and Ruf, W. (2014). Synergies of phosphatidylserine and protein disulfide isomerase in tissue factor activation. Thromb. Haemost. 111, 590-597. doi: 10.1160/TH13-09-0802

Langer, F., Spath, B., Fischer, C., Stolz, M., Ayuk, F. A., Kroger, N., et al. (2013). Rapid activation of monocyte tissue factor by antithymocyte globulin is dependent on complement and protein disulfide isomerase. Blood 121, 2324-2335. doi: 10.1182/blood-2012-10-460493

Lappi, A. K., and Ruddock, L. W. (2011). Reexamination of the role of interplay between glutathione and protein disulfide isomerase. J. Mol. Biol. 409, 238-249. doi: 10.1016/j.jmb.2011.03.024

Laurindo, F. R., Araujo, T. L., and Abrahao, T. B. (2014). Nox NADPH oxidases and the endoplasmic reticulum. Antioxid. Redox Signal. 20, 2755-2775. doi: 10.1089/ars.2013.5605

Laurindo, F. R., Fernandes, D. C., Amanso, A. M., Lopes, L. R., and Santos, C. X. (2008). Novel role of protein disulfide isomerase in the regulation of NADPH oxidase activity: pathophysiological implications in vascular diseases. Antioxid. Redox Signal. 10, 1101-1113. doi: 10.1089/ars.2007.2011

Laurindo, F. R., Pescatore, L. A., and Fernandes Dde, C. (2012). Protein disulfide isomerase in redox cell signaling and homeostasis. Free Radic. Biol. Med. 52, 1954-1969. doi: 10.1016/j.freeradbiomed.2012.02.037

Lopez, J. A., Leung, B., Reynolds, C. C., Li, C. Q., and Fox, J. E. (1992). Efficient plasma membrane expression of a functional platelet glycoprotein Ib-IX complex requires the presence of its three subunits. J. Biol. Chem. 267, 12851-12859.

Ma, Y. Q., Qin, J., and Plow, E. F. (2007). Platelet integrin alpha(IIb)beta(3): activation mechanisms. J. Thromb. Haemost. 5, 1345-1352. doi: 10.1111/j.15387836.2007.02537.x

Maattanen, P., Gehring, K., Bergeron, J. J., and Thomas, D. Y. (2010). Protein quality control in the ER: the recognition of misfolded proteins. Semin. Cell Dev. Biol. 21, 500-511. doi: 10.1016/j.semcdb.2010.03.006

Marchetti, P., Bugliani, M., Lupi, R., Marselli, L., Masini, M., Boggi, U., et al. (2007). The endoplasmic reticulum in pancreatic beta cells of type 2 diabetes patients. Diabetologia 50, 2486-2494. doi: 10.1007/s00125-007-0816-8

Marcus, N., Shaffer, D., Farrar, P., and Green, M. (1996). Tissue distribution of three members of the murine protein disulfide isomerase (PDI) family. Biochim. Biophys. Acta 1309, 253-260.

Mares, R. E., Melendez-Lopez, S. G., and Ramos, M. A. (2011). Acid-denatured Green Fluorescent Protein (GFP) as model substrate to study the chaperone activity of protein disulfide isomerase. Int. J. Mol. Sci. 12, 4625-4636. doi: 10.3390/ijms12074625

Masui, S., Vavassori, S., Fagioli, C., Sitia, R., and Inaba, K. (2011). Molecular bases of cyclic and specific disulfide interchange between human ERO1alpha protein and protein-disulfide isomerase (PDI). J. Biol. Chem. 286, 16261-16271. doi: 10.1074/jbc.M111.231357

McLaughlin, S. H., and Bulleid, N. J. (1998). Thiol-independent interaction of protein disulphide isomerase with type $\mathrm{X}$ collagen during intra-cellular folding and assembly. Biochem. J. 331(Pt 3), 793-800.

Modderman, P. W., Admiraal, L. G., Sonnenberg, A., and Von Dem Borne, A. E. (1992). Glycoproteins V and Ib-IX form a noncovalent complex in the platelet membrane. J. Biol. Chem. 267, 364-369.

Monroe, D. M., Hoffman, M., and Roberts, H. R. (2002). Platelets and thrombin generation. Arterioscler. Thromb. Vasc. Biol. 22, 1381-1389. doi: 10.1161/01.ATV.0000031340.68494.34

Murphy, D. D., Reddy, E. C., Moran, N., and O'neill, S. (2014). Regulation of platelet activity in a changing redox environment. Antioxid. Redox Signal. 20, 2074-2089. doi: 10.1089/ars.2013.5698

Nguyen, V. D., Saaranen, M. J., Karala, A. R., Lappi, A. K., Wang, L., Raykhel, I. B., et al. (2011). Two endoplasmic reticulum PDI peroxidases increase the efficiency of the use of peroxide during disulfide bond formation. J. Mol. Biol. 406 503-515. doi: 10.1016/j.jmb.2010.12.039

Noiva, R. (1999). Protein disulfide isomerase: the multifunctional redox chaperone of the endoplasmic reticulum. Semin. Cell Dev. Biol. 10, 481-493. doi: 10.1006/scdb.1999.0319

Pescatore, L. A., Bonatto, D., Forti, F. L., Sadok, A., Kovacic, H., and Laurindo, F. R. (2012). Protein disulfide isomerase is required for platelet-derived growth factor-induced vascular smooth muscle cell migration, Nox1 NADPH oxidase expression, and RhoGTPase activation. J. Biol. Chem. 287, 29290-29300. doi: 10.1074/jbc.M112.394551

Popescu, N. I., Lupu, C., and Lupu, F. (2010). Extracellular protein disulfide isomerase regulates coagulation on endothelial cells through modulation of phosphatidylserine exposure. Blood 116, 993-1001. doi: 10.1182/blood-200910-249607

Purvis, J. E., Chatterjee, M. S., Brass, L. F., and Diamond, S. L. (2008). A molecular signaling model of platelet phosphoinositide and calcium regulation during homeostasis and P2Y1 activation. Blood 112, 4069-4079. doi: 10.1182/blood2008-05-157883

Ramachandran, N., Root, P., Jiang, X. M., Hogg, P. J., and Mutus, B. (2001). Mechanism of transfer of NO from extracellular S-nitrosothiols into the cytosol by cell-surface protein disulfide isomerase. Proc. Natl. Acad. Sci. U.S.A. 98, 9539-9544. doi: 10.1073/pnas.171180998

Reiser, K., Francois, K. O., Schols, D., Bergman, T., Jornvall, H., Balzarini, J., et al. (2012). Thioredoxin-1 and protein disulfide isomerase catalyze the reduction of similar disulfides in HIV gp120. Int. J. Biochem. Cell Biol. 44, 556-562. doi: 10.1016/j.biocel.2011.12.015

Root, P., Sliskovic, I., and Mutus, B. (2004). Platelet cell-surface protein disulphide-isomerase mediated S-nitrosoglutathione consumption. Biochem. J. 382, 575-580. doi: 10.1042/BJ20040759

Roth, R. A. (1981). Bacitracin: an inhibitor of the insulin degrading activity of glutathione-insulin transhydrogenase. Biochem. Biophys. Res. Commun. 98, 431-438.

Ruggeri, Z. M. (2007). The role of von Willebrand factor in thrombus formation. Thromb. Res. 120(Suppl. 1), S5-S9. doi: 10.1016/j.thromres.2007.03.011

Ryser, H. J., Levy, E. M., Mandel, R., and Disciullo, G. J. (1994). Inhibition of human immunodeficiency virus infection by agents that interfere with thiol-disulfide interchange upon virus-receptor interaction. Proc. Natl. Acad. Sci. U.S.A. 91, 4559-4563.

Santos, C. X., Stolf, B. S., Takemoto, P. V., Amanso, A. M., Lopes, L. R., Souza, E. B., et al. (2009a). Protein disulfide isomerase (PDI) associates with NADPH oxidase and is required for phagocytosis of Leishmania chagasi promastigotes by macrophages. J. Leukoc. Biol. 86, 989-998. doi: 10.1189/jlb. 0608354

Santos, C. X., Tanaka, L. Y., Wosniak, J., and Laurindo, F. R. (2009b). Mechanisms and implications of reactive oxygen species generation during the unfolded protein response: roles of endoplasmic reticulum oxidoreductases, mitochondrial electron transport, and NADPH oxidase. Antioxid. Redox Signal. 11, 2409-2427. doi: 10.1089/ARS.2009.2625

Schulman, S., Wang, B., Li, W., and Rapoport, T. A. (2010). Vitamin K epoxide reductase prefers ER membrane-anchored thioredoxin-like redox partners. Proc. Natl. Acad. Sci. U.S.A. 107, 15027-15032. doi: 10.1073/pnas.1009 972107

Shao, F., Bader, M. W., Jakob, U., and Bardwell, J. C. (2000). DsbG, a protein disulfide isomerase with chaperone activity. J. Biol. Chem. 275, 13349-13352. doi: 10.1074/jbc.275.18.13349

Sliskovic, I., Raturi, A., and Mutus, B. (2005). Characterization of the Sdenitrosation activity of protein disulfide isomerase. J. Biol. Chem. 280, 8733-8741. doi: 10.1074/jbc.M408080200

Sobierajska, K., Skurzynski, S., Stasiak, M., Kryczka, J., Cierniewski, C. S., and Swiatkowska, M. (2014). Protein disulfide isomerase directly interacts with betaactin Cys374 and regulates cytoskeleton reorganization. J. Biol. Chem. 289, 5758-5773. doi: 10.1074/jbc.M113.479477

Taylor, M., Banerjee, T., Ray, S., Tatulian, S. A., and Teter, K. (2011). Proteindisulfide isomerase displaces the cholera toxin Al subunit from the holotoxin without unfolding the Al subunit. J. Biol. Chem. 286, 22090-22100. doi: 10.1074/jbc.M111.237966

Taylor, M., Burress, H., Banerjee, T., Ray, S., Curtis, D., Tatulian, S. A., et al. (2014). Substrate-induced unfolding of protein disulfide isomerase displaces the cholera toxin Al subunit from its holotoxin. PLoS Pathog. 10:e1003925. doi: 10.1371/journal.ppat.1003925

Walker, K. W., and Gilbert, H. F. (1997). Scanning and escape during proteindisulfide isomerase-assisted protein folding. J. Biol. Chem. 272, 8845-8848.

Wan, S. W., Lin, C. F., Lu, Y. T., Lei, H. Y., Anderson, R., and Lin, Y. S. (2012). Endothelial cell surface expression of protein disulfide isomerase activates betal and beta 3 integrins and facilitates dengue virus infection. J. Cell. Biochem. 113, 1681-1691. doi: 10.1002/jcb.24037 
Wang, C., Li, W., Ren, J., Fang, J., Ke, H., Gong, W., et al. (2013). Structural insights into the redox-regulated dynamic conformations of human protein disulfide isomerase. Antioxid. Redox Signal. 19, 36-45. doi: 10.1089/ars.2012.4630

Wang, C., Yu, J., Huo, L., Wang, L., Feng, W., and Wang, C. C. (2012). Human protein-disulfide isomerase is a redox-regulated chaperone activated by oxidation of domain a. J. Biol. Chem. 287, 1139-1149. doi: 10.1074/jbc.M111.303149

Wang, L., Zhang, L., Niu, Y., Sitia, R., and Wang, C. C. (2014). Glutathione peroxidase 7 utilizes hydrogen peroxide generated by Erolalpha to promote oxidative protein folding. Antioxid. Redox Signal. 20, 545-556. doi: 10.1089/ars. 2013.5236

Wang, L., Zhu, L., and Wang, C. C. (2011). The endoplasmic reticulum sulfhydryl oxidase Erolbeta drives efficient oxidative protein folding with loose regulation. Biochem. J. 434, 113-121. doi: 10.1042/BJ20101357

Xu, S., Butkevich, A. N., Yamada, R., Zhou, Y., Debnath, B., Duncan, R., et al. (2012). Discovery of an orally active small-molecule irreversible inhibitor of protein disulfide isomerase for ovarian cancer treatment. Proc. Natl. Acad. Sci. U.S.A. 109, 16348-16353. doi: 10.1073/pnas.1205226109

Yoshimori, T., Semba, T., Takemoto, H., Akagi, S., Yamamoto, A., and Tashiro, Y. (1990). Protein disulfide-isomerase in rat exocrine pancreatic cells is exported from the endoplasmic reticulum despite possessing the retention signal. J. Biol. Chem. 265, 15984-15990.

Conflict of Interest Statement: The authors declare that the research was conducted in the absence of any commercial or financial relationships that could be construed as a potential conflict of interest.

Received: 03 June 2014; accepted: 07 August 2014; published online: 26 August 2014. Citation: Ali Khan H and Mutus B (2014) Protein disulfide isomerase a multifunctional protein with multiple physiological roles. Front. Chem. 2:70. doi: 10.3389/ fchem.2014.00070

This article was submitted to Cellular Biochemistry, a section of the journal Frontiers in Chemistry.

Copyright (c) 2014 Ali Khan and Mutus. This is an open-access article distributed under the terms of the Creative Commons Attribution License (CC BY). The use, distribution or reproduction in other forums is permitted, provided the original author(s) or licensor are credited and that the original publication in this journal is cited, in accordance with accepted academic practice. No use, distribution or reproduction is permitted which does not comply with these terms. 